\title{
Metodologia para estimar a estabilidade do conjunto muda x substrato de cafeeiro
}

\author{
A methodology to evaluate the stability of coffee seedling
}

\author{
José Laércio Favarin I José Laércio Favarin Junior ${ }^{\mathrm{II}}$ André Rodrigues dos Reis ${ }^{\mathrm{III}}$ \\ Fabiana Taveira Camargo III
}

\section{RESUMO}

A utilização de substrato em tubetes para a formação de mudas de café aumentou em substituição à mistura de solo argiloso com resíduo orgânico, resistente à deformação da muda no plantio, devido à coesão e aderência às raízes. Por sua vez, o substrato em tubetes esboroa com facilidade, em razão da granulometria do material. Do exposto, avaliouse uma metodologia para estimar a estabilidade ao manuseio do conjunto muda $x$ substrato, com base na variação granulométrica (G1: 30,86 \%; G2: 62,26\% e G3: 49,83\% de partículas nas peneiras 0,50 a $0,05 \mathrm{~mm}$ ) e do volume de material (V1: 50, V2: 120 e V3: 200 $\mathrm{cm}^{3}$ ), em mudas de café Catuaí Vermelho IAC 144 com quatro pares de folhas. A estabilidade do conjunto muda $x$ substrato foi estimada pela quantidade de substrato desagregado e partes de raízes, após submetê-lo a cinco ciclos de 60 segundos de rotação (1.750rpm) em mesa vibratória. A estabilidade foi maior nos tubetes de 50 e $120 \mathrm{~cm}^{3}$ de substrato. A redução parcial da granulometria (50\% substrato na granulometria original e 50\% substrato moído) aumentou a aderência das partículas às raízes e a estabilidade do conjunto nos recipientes menores.

Palavras-chave: Coffea arabica L., tubetes, propagação, mesa vibratória.

\section{ABSTRACT}

The objectives of this research are the evaluation of substrate volume and granulometer influence - used in production of coffee seedlings in plastic tubes - on coffee plants growth, the time of seedlings development and also seedlingsubstrate handling stability. The investigation was carried out in a nursery at Coffee Experimental Center of IAC, SP, Brazil, with the cultivar Catuaí Vermelho IAC 144 (Coffea arabica L.). Nine treatments were tested with four replicates and the experimental design used was randomized blocks with $3 \times 3$ factorial composed by three substrate volumes (V1: 50, V2: 120 and V3: $200 \mathrm{~cm}^{3}$ ) and three granulometer substrate levels (G1: $30.86 \%, G 2: 62.26 \%$ and G3: $49.83 \%$ of particles in sieves 0.50 to $0.05 \mathrm{~mm}$ ). The stability of seedlings to handle was evaluated using a vibratory table, with basis of amount of crumbling material (substrate and roots). The joint was submitted to rotation during 60 seconds, repeated five times (5 cycles). The end of each cycle the unfastened material was collected and definitive to disaggregated mass. The stability of joint seedling-substrate varied with the size of container, bigger in tubetes with $50 \mathrm{~cm}^{3}$ and $120 \mathrm{~cm}^{3}$ of substrate. The partial reduction of granulometry (50\% of substrate on original granulometer and $50 \%$ of crushed substrate) increased the adhesion of particles with roots and, the stability of joint.

Key words: Coffea arabica L., conic plastic tubes, propagation, vibratory table.

\section{INTRODUÇÃO}

A granulometria é um atributo físico muito importante, que não tem recebido a mesma ênfase que os atributos químicos nas pesquisas com substrato Esta característica é fundamental à manutenção da integridade do conjunto muda $\mathrm{x}$ substrato e à preservação da sua estabilidade após a retirada do tubete e o manuseio da muda no plantio. Tal fato é desejável, pois as raízes de cafeeiro são pouco lignificadas e, portanto, suscetíveis às rupturas e aos desvios laterais.

Estudos sobre a influência do substrato sobre o crescimento de mudas de essências florestais

'Departamento de Produção Vegetal, Escola Superior de Agricultura Luiz de Queiroz (ESALQ), Universidade de São Paulo (USP),

13418-900, Piracicaba, SP, Brasil. E-mail: jlfavari@esalq.usp.br. Autor para correspondência.

${ }^{\text {IIC }}$ urso de Agronomia, Universidade Federal de Lavras (UFLA), Lavras, MG, Brasil.

IIIPrograma de Pós-graduação em Agronomia, ESALQ/USP, Piracicaba, SP, Brasil 
em tubetes realizadas por PHIPPS (1974) indicam que a composição pode afetar tanto o crescimento quanto a sobrevivência das plantas, não sendo, no entanto, as diferenças atribuídas, exclusivamente, aos atributos químicos do material. A importância dos aspectos físicos do substrato, na formação de mudas de cafeeiro, também foi comprovada pela pesquisa desenvolvida porTAVARES JÚNIOR(2004).

A maioria dos trabalhos realizados com substratos comerciais na formação de mudas de cafeeiro enfoca, predominantemente, aspectos químicos do material, como a dose a ser aplicada, sua composição em nutrientes, a solubilidade da fonte e o tempo de liberação dos nutrientes (FAVORETO et al., 1998; MELO, 1999; MELO et al., 1999). Não há na literatura uma metodologia para estimar a estabilidade do conjunto muda x substrato, a qual é fundamental para reduzir o risco de dano às raízes e facilitar o pegamento da muda após o transplante no campo.

A granulometria do material influencia a aeração às raízes, mas não tem sido avaliada nos trabalhos dessa natureza. Pode-se admitir, por hipótese, que a aderência entre as partículas do substrato com as raízes depende da textura do material. No sistema tradicional de formação de mudas de cafeeiro, utilizase a mistura de solo argiloso com resíduos orgânicos, que resulta num material muito coeso e aderido às raízes, cuja resistência reduz a deformação após a retirada do recipiente. Assim, as raízes não são expostas às pressões feitas para eliminar os espaços vazios entre a muda e o solo, preserva-se a disposição radicular nos poros do substrato e diminuiem-se os danos radiculares, bem como o estresse das plantas, o que resulta em maior número de plantas sobreviventes, além do reinício antecipado do crescimento do vegetal após o transplante.

Assumiu-se, por hipótese, que a redução da granulometria do substrato aumentaria a adesão das partículas às raízes das plantas e, conseqüentemente, a resistência ao manuseio do conjunto muda $x$ substrato, preservando a sua estabilidade após a retirada do tubete para o plantio.

Este trabalho foi realizado com o objetivo de avaliar uma metodologia para estimar quantitativamente a estabilidade ao manuseio do conjunto muda x substrato, com base na variação da granulometria e do volume de material utilizado na produção de mudas de cafeeiro em tubetes.

\section{MATERIAL E MÉTODOS}

O experimento foi instalado no viveiro de mudas do Centro de café e plantas tropicais do Instituto
Agronômico, na Fazenda Santa Elisa, Campinas, SP, utilizando-se sementes de Catuaí Vermelho IAC 144 semeadas na proporção de $1,0 \mathrm{~kg} \mathrm{~m}^{-2}$, em germinadores de areia. As plântulas, no estádio de folhas cotiledonares ("orelha de onça”), após a retirada do terço final da raiz principal, foram transplantadas em tubetes.

Os tratamentos foram obtidos pela combinação de três volumes de substrato (V1: 50; V2: 120 e V3: $200 \mathrm{~cm}^{3}$ ) e três classes granulométricas (G1: 30,86\%; G2: 62,26\% e G3: 49,83\% de partículas nas peneiras 0,50 a $0,05 \mathrm{~mm})$, a partir da granulometria original do substrato comercial (SC) (Tabela 1), instalados em blocos ao acaso em esquema fatorial, com quatro repetições. A escolha dos volumes de substratos foi feita usando-se o recipiente padrão (TAVARES JÚNIOR, 2004) na formação de mudas de cafeeiro em tubetes $\left(120 \mathrm{~cm}^{3}\right)$, acrescentando-se recipientes com capacidade inferior $\left(50 \mathrm{~cm}^{3}\right)$ e com capacidade superior $\left(200 \mathrm{~cm}^{3}\right)$.

Nos tratamentos V1G1, V2G1 e V3G1, foi usado o substrato comercial plantmax ${ }^{\circledR}$, composto pela mistura de vermiculita expandida, casca de pínus, turfa e perlita, que, na granulometria original (G1 - SC), era constituído por $30,86 \%$ das partículas retidas nas peneiras 0,50 a 0,05mm. Nos tratamentos V1G2, V2G2 e V3G2, foi utilizado o mesmo substrato após a moagem (G2 - SM), apresentando 62,26\% das partículas retidas nas peneiras 0,50 a $0,05 \mathrm{~mm}$, praticamente o dobro em relação à granulometria original. Os tratamentos V1G3, V2G3 e V3G3 obtidos pela mistura, em volume, de partes iguais entre ambos (G3 - SC+SM), continham 49,83\% das partículas retidas nas peneiras 0,50 a $0,05 \mathrm{~mm}$, portanto, com composição intermediária entre os tratamentos anteriores (G1: 30,86\% e G2: 62,26\%).

O substrato fino (SM) foi obtido pela moagem do produto comercial (SC) em moinho de martelo, em que o material foi pressionado contra uma peneira instalada na saída do equipamento. Do material moído, recolheu-se uma parte, que foi submetida a um conjunto de peneiras para a separação e identificação das composições granulométricas (G1, G2 e G3).

A adubação do substrato foi feita com uma dose de $18 \mathrm{~g} \mathrm{~kg}^{-1}$ de substrato do fertilizante osmocote contendo 15: 10: 10 (\% de $\mathrm{N}, \mathrm{P}_{2} \mathrm{O}_{5}$ e $\mathrm{K}_{2} \mathrm{O}$ ), além de 3,5\% de Ca; $1,5 \%$ de Mg; 3,0\% de S; 0,05\% de Zn; 0,02\% de B; 0,05\% de Cu; 0,1\% de Mn; 0,5\% de Fe e 0,004\% de Mo. Durante a condução do experimento, foram efetuadas três adubações complementares, via fertirrigação, utilizando-se 2,0g do fertilizante 15:5:15 (\% de $\mathrm{N}, \mathrm{P}_{2} \mathrm{O}_{5}$ e $\mathrm{K}_{2} \mathrm{O}$ ) por litro de água, aplicados a cada 30 dias a partir do lançamento do segundo par de folhas até o quarto par de folhas. 
Tabela 1 - Tratamentos com base no volume e na composição granulométrica do material a partir da granulometria original do substrato plantmax ${ }^{\circledR}$.

\begin{tabular}{ccl}
\hline Tratamentos & Volume $\left(\mathrm{cm}^{3}\right)$ & \multicolumn{1}{c}{ Composição granulométrica do substrato } \\
\hline V1G1 & 50 & $100 \%$ substrato na granulometria original (SC) \\
V1G2 & 50 & $100 \%$ substrato finamente moído (SM) \\
V1G3 & 50 & $50 \%$ substrato (SC) + 50\% substrato moído (SM) \\
V2G1 & 120 & $100 \%$ substrato na granulometria original (SC) \\
V2G2 & 120 & $100 \%$ substrato finamente moído (SM) \\
V2G3 & 120 & $50 \%$ substrato (SC) + 50\% substrato moído (SM) \\
V3G1 & 200 & $100 \%$ substrato na granulometria original (SC) \\
V3G2 & 200 & $100 \%$ substrato finamente moído (SM) \\
V3G3 & 200 & $50 \%$ substrato (SC) + 50\% substrato moído (SM) \\
\hline
\end{tabular}

${ }^{1}$ mistura granulométrica com base em volume.

De cada repetição, foram utilizadas 10 plantas, ao acaso, para as determinações do comprimento de raízes, superfície radicular, matéria seca de raízes, e a estimativa da estabilidade do conjunto muda $\mathrm{x}$ substrato.

A superfície radicular (SR) e o comprimento das raízes (CR) foram obtidos por meio da captura de imagens de raízes dispostas sobre um scanner de mesa e processadas pelo software SIARCS 3.0 (CRUVINEL et al., 1996). A matéria seca de raízes (em g) foi obtida após a secagem do material em estufa com circulação de ar forçada a $70^{\circ} \mathrm{C}$ durante 36 horas.

A estabilidade da muda ao manuseio foi avaliada no final do experimento, quando as plantas apresentavam quatro pares de folhas expandidas, o que ocorreu aos 81 dias nos recipientes de $200 \mathrm{~cm}^{3}, 124$ dias nos tubetes de $120 \mathrm{~cm}^{3}$ e 134 dias naqueles de $50 \mathrm{~cm}^{3}$. O teste foi realizado após o corte da parte aérea na região do colo, depois de terem sido mantidas em recipiente com água visando à uniformização da umidade de cada conjunto muda x substrato.

O teste foi efetuado numa mesa vibratória com a finalidade de separar as raízes dos substratos (desagregação), cuja estabilidade foi estimada com base na quantidade de material esboroado (substrato e raízes, em g) durante a operação. Para tanto, foram utilizadas três plantas por parcela e quatro repetições, totalizando 12 plantas por tratamento. A muda foi colocada em becker de $10 \mathrm{~cm}$ de diâmetro, ajustado a uma mesa vibratória e submetido a cinco ciclos de 60 segundos de rotação a $1.750 \mathrm{rpm}$, e, ao final de cada ciclo, recolheu-se e determinou-se a matéria fresca (substrato e raízes) do material desprendido.

Os resultados obtidos foram submetidos à análise de variância e, ao ser constatada a significância pelo teste $\mathrm{F}$, compararam-se as médias dos tratamentos por meio do teste Tukey a 5\% de probabilidade de erro.

\section{RESULTADOS E DISCUSSÃo}

A quantidade de material desagregado, independentemente do ciclo de vibração, foi inferior para o conjunto muda $x$ substrato dos recipientes de 50 e $120 \mathrm{~cm}^{3}$, comparada àquela determinada nos tubetes de $200 \mathrm{~cm}^{3}$ (Tabela 2). A matéria seca, o comprimento e a superfície das raízes foram menores nas plantas dos tubetes de 50 e $120 \mathrm{~cm}^{3}$ (Tabela 3). Nestes conjuntos (muda x substrato), houve resistência superior ao

Tabela 2 - Massa de substrato e raízes desagregados do cafeeiro do conjunto muda-substrato ao final de cada ciclo de 60 segundos de vibração, em razão do volume do substrato.

\begin{tabular}{|c|c|c|c|c|c|}
\hline \multirow{2}{*}{ Volume de substrato $\left(\mathrm{cm}^{3}\right)$} & \multicolumn{5}{|c|}{ Ciclos de vibração (60 s) } \\
\hline & $1^{\circ}$ ciclo & $2^{\circ}$ ciclo & $3^{\circ}$ ciclo & $4^{\circ}$ ciclo & $5^{\circ}$ ciclo \\
\hline & \multicolumn{5}{|c|}{ Massa de substrato e raízes (grama) } \\
\hline 50 & $29,21 b$ & $29,59 b$ & $31,10 \mathrm{~b}$ & $32,10 \mathrm{~b}$ & $34,29 b$ \\
\hline 120 & $26,13 b$ & $30,45 b$ & $32,16 b$ & $32,74 b$ & $35,09 b$ \\
\hline 200 & $34,25 a$ & $37,98 a$ & $41,34 a$ & 44,18a & $47,38 \mathrm{a}$ \\
\hline CV (\%) & 19,42 & 3,73 & 2,65 & 4,20 & 2,31 \\
\hline
\end{tabular}

Médias não seguidas pelas mesmas letras nas colunas diferem entre si pelo teste Tukey a 5\% de probabilidade de erro.

Ciência Rural, v.38, n.1, jan-fev, 2008. 
Tabela 3 - Matéria seca de raízes (MSR), superfície radicular (SR) e comprimento das raízes (CR) das mudas de cafeeiro em razão do volume do substrato.

\begin{tabular}{cccc}
\hline Volume de substrato $\left(\mathrm{cm}^{3}\right)$ & Matéria seca de raízes $(\mathrm{g})$ & Superfície radicular $\left(\mathrm{cm}^{2}\right)$ & Comprimento de raízes $(\mathrm{cm})$ \\
\hline 50 & $0,46 \mathrm{c}$ & $85,59 \mathrm{c}$ & $14,93 \mathrm{c}$ \\
120 & $0,77 \mathrm{~b}$ & $114,10 \mathrm{~b}$ & $21,07 \mathrm{~b}$ \\
200 & $0,90 \mathrm{a}$ & $166,27 \mathrm{a}$ & $31,43 \mathrm{a}$ \\
$\mathrm{CV}(\%)$ & 10,03 & 20,63 & 13,28 \\
\hline
\end{tabular}

Médias não seguidas pelas mesmas letras na linha diferem entre si pelo teste Tukey a 5 \% de probabilidade de erro.

esboroamento, pela aderência entre as raízes e as partículas do material, em razão do menor volume de substrato.

A condução de mudas em menor volume de substrato implica, provavelmente, no aumento da dificuldade de as raízes ocuparem os espaços no solo adjacente, após o plantio no campo, confinando o sistema radicular, por mais tempo, a um pequeno volume de solo. Se esta hipótese for comprovada, a retomada do crescimento da muda seria prejudicada, bem como o crescimento e o estabelecimento inicial das plantas, o que implicaria a necessidade de replantios. Por outro lado, a integridade do sistema radicular é mais bem preservada nos tubetes de menor volume e mais econômico pelo uso de menos substrato.

A constatação de menor estabilidade do conjunto em que se usou maior volume de substrato $\left(200 \mathrm{~cm}^{3}\right)$ pode ser uma conseqüência dos atributos radiculares (comprimento e superfície) na fase de quatro pares de folhas (época da avaliação). Neste estádio, os atributos radiculares não foram suficientes para promover a aderência necessária e, consequentemente, manter a integridade do conjunto, em razão do maior volume de material. Assim, sugere-se que as mudas de cafeeiro formadas em recipientes sejam levadas ao campo em estádio que apresente mais de quatro pares de folhas (Tabela 2).

A estabilidade das mudas ao manuseio não foi influenciada pela granulometria no primeiro ciclo vibratório (Tabela 4). Entretanto, a partir do segundo ciclo, foram constatados dois níveis de estabilidades, observando-se menor resistência dos conjuntos provenientes do substrato moído (G2 - 100\% SM), como indica o aumento da quantidade de material esboroado e de partes de raízes $(35,14 \mathrm{~g})$. A partir do terceiro ciclo, puderam ser diferenciadas as três composições granulométricas em relação à estabilidade, em que o maior esboroamento ocorreu com o conjunto proveniente da granulometria moída (G2 - SM: 40,12g), seguida das mudas formadas na granulometria original (G1 - SC: 33,20g) e, posteriormente, daquelas da mistura granulométrica (G3-SC + SM: 31,26g).

No terceiro, quarto e quinto ciclos vibratórios, a mistura granulométrica (G3 - SC + SM) apresentou a menor quantidade de material esboroado (substrato e partes de raízes), o que implica maior estabilidade do conjunto muda x substrato. Portanto, a diminuição da granulometria foi benéfica, confirmando, em parte, a hipótese deste trabalho, de que a redução do tamanho das partículas aumentaria a aderência das mesmas às raízes.

Para avaliar a interação entre o volume e a granulometria do substrato, foi apresentada somente a análise da quantidade de material desagregado no terceiro ciclo de vibração, quando, efetivamente, foram distinguidas, pela primeira vez, as diferenças entre as composições granulométricas em relação à estabilidade (Tabela 5). A combinação entre o menor volume de

Tabela 4 - Massa de substrato e raízes desagregados do conjunto muda-substrato de cafeeiro ao final de cada ciclo de 60 segundos de vibração em razão da composição granulométrica do substrato.

\begin{tabular}{|c|c|c|c|c|c|}
\hline \multirow{2}{*}{ Composição granulométrica do substrato } & \multicolumn{5}{|c|}{ Ciclos de vibração (60s) } \\
\hline & $1^{\mathrm{o}}$ ciclo & $2^{o}$ ciclo & $3^{\circ}$ ciclo & $4^{\underline{0}}$ ciclo & $5^{\circ}$ ciclo \\
\hline & \multicolumn{5}{|c|}{ Massa de substrato e raízes (grama) } \\
\hline $100 \%$ substrato comercial (SC) & $30,60 \mathrm{a}$ & $31,72 b$ & 33,20b & $34,40 b$ & $36,59 b$ \\
\hline 100\% substrato moído (SM) & $32,34 a$ & $35,14 a$ & $40,12 \mathrm{a}$ & $42,45 a$ & $45,79 a$ \\
\hline $50 \% \mathrm{SC}+50 \% \mathrm{SM}$ & $26,66 a$ & $31,14 b$ & $31,26 c$ & $32,15 c$ & $34,38 c$ \\
\hline CV (\%) & 19,42 & 3,73 & 2,65 & 4,20 & 2,31 \\
\hline
\end{tabular}

Médias não seguidas pelas mesmas letras nas colunas diferem entre si pelo teste Tukey a 5\% de probabilidade de erro. 
Tabela 5 - Massa de substrato e raízes desagregados do conjunto muda-substrato de cafeeiro ao final do terceiro ciclo de 60 segundos de vibração em razão da interação entre o volume e a composição granulométrica do substrato.

\begin{tabular}{|c|c|c|c|c|}
\hline \multirow{2}{*}{ Volume de substrato } & \multicolumn{4}{|c|}{ Composição granulométrica do substrato } \\
\hline & $100 \%$ substrato comercial (SC) & 100\% substrato moído (SM) & $50 \% \mathrm{SC}+50 \% \mathrm{SM}$ & Médias \\
\hline & \multicolumn{4}{|c|}{ Massa de substrato e raízes (grama) } \\
\hline $50 \mathrm{~cm}^{-3}$ & $33,63 \mathrm{aA}$ & $31,10 \mathrm{bB}$ & $28,57 b C$ & 31,10 \\
\hline $120 \mathrm{~cm}^{-3}$ & $32,07 \mathrm{aA}$ & $32,43 \mathrm{bA}$ & $31,97 a A$ & 32,15 \\
\hline $200 \mathrm{~cm}^{-3}$ & $33,90 \mathrm{aB}$ & $56,83 a A$ & $33,27 \mathrm{aB}$ & 41,33 \\
\hline Médias & 33,20 & 40,12 & 31,27 & 34,33 \\
\hline CV (\%) & - & - & - & 19,42 \\
\hline
\end{tabular}

Médias seguidas pelas mesmas letras minúsculas na coluna e maiúsculas na linha não diferem entre si pelo teste Tukey a 5\% de probabilidade de erro.

substrato $\left(50 \mathrm{~cm}^{3}\right)$ com a redução do tamanho das partículas influenciou a estabilidade do conjunto de acordo com a seguinte ordem decrescente: mistura granulométrica (G3 - SC + SM: 28,57g), substrato moído (G2 - SM: 31,10g) e substrato na granulometria comercial (G1 - SC: 33,63g).

Para o recipiente comumente utilizado na formação de mudas de cafeeiro $\left(120 \mathrm{~cm}^{3}\right)$, não foi constatada influência da granulometria na estabilidade do conjunto. Por sua vez, com o maior volume de substrato moído $\left(200 \mathrm{~cm}^{3}\right)$, aumentou a desagregação (G2 - SM: 56,83g), em relação às demais composições granulométricas (G1 - SC: 33,90g) e (G3 - SC + SM: $33,27 g$ ), não confirmando, em parte, a hipótese de que a redução do tamanho das partículas promoveria a estabilidade. A quantidade de substrato pode ser um entrave à obtenção da estabilidade do conjunto, porque a aderência entre as partes (raízes e substrato) teria que acontecer com um maior volume de partículas. Portanto, pode-se afirmar que, em mudas mais velhas, em relação àquelas usadas nesta pesquisa (quatro pares de folhas), poder-se-ia esperar um aumento de raízes e, possivelmente, na aderência do conjunto.

Esta metodologia foi avaliada para estimar quantitativamente a estabilidade do conjunto muda substrato, cuja eficiência depende do ajuste do diâmetro do recipiente preso à mesa vibratória, conforme o tamanho do referido conjunto (muda x substrato), visando à uniformização dos impactos, bem como para evitar danos às mudas durante a retirada do recipiente, para não intensificar a desagregação durante o teste. Ressalta-se que o critério de número de pares de folhas não deve ser utilizado em ensaios dessa natureza, pois há diferenças nos atributos radiculares (comprimento e superfície) de acordo com os volumes de substrato utilizados (50, 120 e $\left.200 \mathrm{~cm}^{3}\right)$. Esse critério pode ser adotado desde que sejam feitas pesquisas para cada volume de substrato isoladamente.

\section{CONCLUSÕES}

A estabilidade do conjunto muda $x$ substrato variou com o tamanho do recipiente, aumentando nos recipientes menores $\left(50\right.$ e $\left.120 \mathrm{~cm}^{3}\right)$ de substrato. A menor estabilidade ocorreu no recipiente de maior volume $\left(200 \mathrm{~cm}^{3}\right)$.

A redução parcial da granulometria, pela mistura de $50 \%$ de substrato na granulometria original e de $50 \%$ de substrato moído, aumentou a aderência das partículas às raízes e a estabilidade do conjunto nos recipientes menores $\left(50\right.$ e $\left.120 \mathrm{~cm}^{3}\right)$.

\section{REFERÊNCIAS}

CRUVINEL, P.E. et al. Métodos e aplicações do processamento de imagens digitais. In: __. Instrumentação agropecuária: contribuições no limiar do novo século. Brasília: EMBRAPA-SPI, 1996. Cap.3, p.91-151.

FAVORETO, A.J. et al. Uso de fontes orgânicas e fertilizantes de liberação lenta na formação de mudas de café em sacos de polietileno. In: CONGRESSO BRASILEIRO DE PESQUISAS CAFEEIRAS, 24., 1998, Poços de Caldas. Anais. Rio de Janeiro: IBG, GERCA, 1998. p.144-145.

MELO, B. Estudos sobre a produção de mudas de cafeeiro (Coffea arabica L.) em tubetes. 1999. 119f. Tese (Doutorado em Agronomia) - Universidade Federal de Lavras.

MELO, B. et al. Substratos, fontes e doses de P2O5 na produção de mudas de cafeeiro (Coffea arabica L.) em tubetes. In: CONGRESSO BRASILEIRO DE PESQUISAS CAFEEIRAS, 25., 1999, Franca. Anais... Rio de Janeiro: IBG, GERCA, 1999. p.176-177.

PHIPPS, H.M. Influence of media on growth and survive all of container - grown seedling. In: NORTH AMERICAN CONTAINERIZER FOREST TREE SEEDLING SYMPOSIUM, 1974, Denver. Proceedings... Denver: Great Plains Agricultural Council, 1974. p.398-400.

TAVARES JÚNIOR, J.E. Volume e granulometria do substrato na formação de mudas de café. 2004. 59f. Dissertação (Mestrado em Agronomia) - Curso de Pós-graduação em Agronomia, Área de concentração em Fitotecnia, Escola Superior de Agricultura “Luiz de Queiroz”, Universidade de São Paulo, Piracicaba.

Ciência Rural, v.38, n.1, jan-fev, 2008. 